\title{
Study of Rheology of Tannin Obtained from Flower Buds of Terminalia chebula (Aralu) and Formaldehyde Reaction
}

\author{
S Arasaretnam \\ Department of Chemistry, Faculty of Science, Eastern University, Sri Lanka \\ *Corresponding Author: S Arasaretnam, Department of Chemistry, Faculty of Science, Eastern \\ University, Sri Lanka

\begin{abstract}
The gelling and hardening reactions of tannin extracted from flower buds of Terminalia chebula with formaldehyde were analysed by parallel-plate rheometry techniques. In this study, a method to determine the rheological features of tannin-formaldehyde reactions both before and after the gel point was settled. The conversion from the liquid to solid state occurs at the gel point. Before the gel point, the zero shear viscosity can portray the behavior of the liquid resin. The value of this parameter rises as the polycondensation reaction proceeds, and at the gel point the weight average molecular mass turn into theoretically infinite.
\end{abstract}

Keywords: gelling, tannin, rheological, viscosity, molar mass

\section{INTRODUCTION}

Commercial polyflavonoid tannin extracts have been produced and used industrially for many applications since the end of the $19^{\text {th }}$ century $[1,2]$. Their use as wood adhesives is based mainly on the reaction, gelling, and hardening of these very reactive, natural phenolic materials with formaldehyde. This is a polycondensation reaction similar but not completely equal to the reaction observed in synthetic phenol-formaldehyde resins [1,2].

Considering the relevance of the rheological behavior of the tannin-formaldehyde reaction on their industrial application as thermosetting wood adhesives, the literature is almost completely devoid of rheological studies on the gelling and hardening of these materials, other than some recent ones using thermo mechanical analysis to follow hardening of the resin in situ on the substrate $[3,4,5,6]$. Although some interesting and valuable information was obtained on the effects of $\mathrm{pH}$ and concentration on the rheological characteristics of various commercial tannins, This study, hence, did not address the rheological characteristics of the formaldehyde induced hardening reaction of tannin extracts of flower buds of Terminalia chebula (Aralu) .

The following study explained the rheological characteristics of the reaction with formaldehyde and tannin extracts from flower buds of Terminalia chebula, extracted under standard conditions.

\section{EXPERIMENTAL TECHNIQUES}

Flower buds of Terminalia chebula were colleted from locally available place in Sri Lanka. The dry flower buds were extracted with hexane, petroleum ether to remove the non-polar compounds from dry buds. Then the flower buds were extracted with ethyl acetate, at the boiling temperature. Non tannin compounds were removed using hexane extraction as much as possible. Finally the extract was concentrated under reduced pressure and the resulting brown precipitate (tannin) was removed from filtration. Phenolic content of the extract was determined using Folin Ciocalteau Colorimetric reaction containing content of $75 \%$ for the ployflavanoid extract. $5 \%$ Para formaldehyde was added to the reaction mixture, with the amount depending on the desired molar ratio of formaldehyde to tannin (F/T). The appropriate amount of $0.1 \mathrm{M} \mathrm{NaOH}$ was added immediately to adjust the $\mathrm{pH}$. The solution were tested with a HAAKE Rotovisco rheometer with cone plate geometry(sensor Z10DIN, mass $37.40 \mathrm{~g}$, radious $5.000 \mathrm{~mm}$, length $15 \mathrm{~mm}$ ) for all the measurements.

The gap in between the cone and parallel plates was $0.43 \mathrm{~mm}$. A humidity cover plus solvent trap based on a film of silicone oil applied to the perimeter of the plates were used to prevent solvent evaporation. 
During rheological measurements, shear was applied sinusoidally at deformation amplitude ( $\gamma$ max) of $5 \%$, hence sufficiently weak not to destroy the structure of the gel or of the network, which forms as the tannin-formaldehyde reactions proceeds.

The measurements were repeated at four different temperatures and at five different frequencies, 2, 4, 6,8 , and $10 \mathrm{rad} / \mathrm{s}$. for the tannin-formaldehyde mixture.

The viscosity at zero frequency $\left(\eta_{0}\right)$ was obtained by the value of the intersection on the $y$-axis. The viscosity extrapolated at zero frequency was used because the viscosity is frequency (i.e., shear rate)dependent. This was done, as explained in several texts [1,2] and clearly stated in one of them [8] so that the gel point can be determined experimentally as a moment when the reactive system loses the possibility to flow. It is evident that this moment depends on the intensity of deformation of the material. Moreover, the higher the shear rate, the earlier the cessation of flow of the curing material was recorded.

A total viscosity profile during gelation can be obtained with the shear rate variation within a few orders of magnitude and the moment of achieving the gel point is determined for the minimum shear rate. For the most exact determination of the gel time by the viscometry method, it is necessary to carry out experiments at several shear rates (i.e., frequencies) with the subsequent extrapolation to the zero rate. [8]

\section{RESULTS AND DISCUSSION}

\subsection{Viscosity as a Function of Time}

In the case of linear polymers, the viscosity is connected to the molecular mass by the expression. [68]

$$
\eta=\kappa^{\prime} M_{n}^{a}
$$

or

$$
\ln \eta=\ln k^{\prime}+a \ln M_{n}
$$

where $\eta$ is the viscosity, $a$ and $c$ are constants, and $M n$ is the molecular mass and where $k^{l}$ is a constant and $a=1$ for $M\langle M c$, or $a=3.4$ for $M>M c$, where $M c$ is the critical mass over which one has a change in the characters of the intra molecular linkages of the polymer.[7]

In the case of a polycondensation reaction, the main reaction phases can be observed by a graph of the $\log$ viscosity as a function of the $\log$ of the reaction time and each linear section of the graph corresponding to a different reaction phase. [9]

As the time increases, the molecular mass increases as the polycondensation goes on, forming longer polymer chains, which means, from eq. (1), that viscosity also increases. In the ideal case in which diffusion problems do not occur, the slope of the straight lines expressing the dependence of the viscosity on the reaction time increases from $1(t<t c)$ to $3.4(t>t c)$, $t c$ being the time when the system reaches the critical mass $M c$. [10,11]

An example is shown in Figure 1 and the value of the slope coefficient $a$ and $b$ obtained for the different poly condensation phases before gelling point are shown in Table 1.

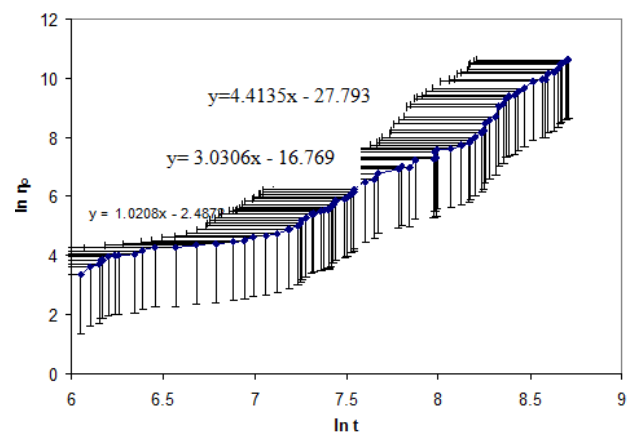

Figure1. Graph in $\ln \eta_{0}$ and $\ln t$ of the increase in viscosity as a function of time of the system aralu tannin extract $+5 \%$ para formaldehyde during hardening at $65^{\circ} \mathrm{C}$ with three linear slopes for the determination of coefficient $a$ and $b$. 
Study of Rheology of Tannin Obtained from Flower Buds of Terminalia chebula (Aralu) and Formaldehyde Reaction

Table1. Coefficients $a$ and $b$ at different temperatures of the Linear Correlation of $\log \left(\eta_{0}\right) \operatorname{Versus} \log (t)$ for the reaction of the tannin extract $+5 \%$ paraformaldehyde, for the three phases of the reaction (see Fig. 1)

\begin{tabular}{|c|c|c|c|c|}
\hline \multicolumn{5}{|c|}{$\log \left(\eta_{0}\right)$ versus $\log (\mathrm{t}) \mathrm{y}=\mathrm{ax}+\mathrm{b}$} \\
\hline \multicolumn{5}{|c|}{ Temperature $\left({ }^{\circ} \mathrm{C}\right)$} \\
\hline Phases & 65 & 70 & 75 & 80 \\
\hline PhaseI & 1.0208 & 1.0903 & 0.7003 & 0.6595 \\
$\mathrm{a}$ & -2.4872 & -3.0892 & -0.8575 & -0.710 \\
b & & & 2.0476 \\
\hline PhaseII & 3.0306 & 2.8789 & 1.8974 & -10.541 \\
a & -16.769 & -15.6160 & -9.3008 & 3.6718 \\
b & 4.4135 & 3.9805 & 3.676 & -23.91 \\
\hline PhaseIII & -27.793 & -24.411 & -23.343 & \\
a &
\end{tabular}

$a$ and $b$ are slope coefficient

The results in Table 1 showed several trends.

(i) An increase in the reaction temperature results in a clear decrease in the value of coefficient $a$ in phase III of the reaction. This means that the degree of polymerization increases more slowly the higher is the reaction temperature. This can be explained by early network immobilization due to the existence of reaction diffusional problems after the gel point. The higher the temperature, the earlier and more marked is early network immobilization and the lower is the slope in the graph.

(ii) With the exception of the $65^{\circ} \mathrm{C}$ case, a similar trend for coefficient $a$ is also noticeable in phase I, but this is due to different reasons. The increase in the limit value $M c$, which is needed for entanglement with an increase in temperature in the early stages of the reaction.

$a \approx 1$ when only small oligomers are present at higher temperature. At lower temperature, the same low mass oligomers give a higher viscosity simply because the limit value $M c$ is lower.

(iii) This is not anymore valid for phase II as a network has already started to form and the increase in the value $a$ of the slope indicates a progressive increase in gel formation with increasing temperature.

(iv) It must also be noted that the scheme as shown in Figure 1, in which the three phases all have slopes higher than the preceding one, is not the most common case (Table 1). In general, the slope of phase II is lower than the preceding one. This means that, in phase II, the increase of the viscosity at zero frequency slows down.

From the experimental results at the kinetics of the tannin-formaldehyde reaction following explanations can be made.

1. As the formation of linear polycondensates advances, it becomes progressively and statistically more difficult to maintain linear chain growth, leading to diffusional control for a chain end to find a chain end and to a slowdown in the increase of the viscosity. The increase in the slope intervening again in phase III corresponds to a change in the type of reaction occurring, namely, it is the phase in which branching starts and where reaction does not need to occur only end to end.

2. This behavior could also stem from two competing reactions characteristic of any phenolformaldehyde resin system. [3,4] In the first networks formed, a considerable number of methylene ether bridges $-\mathrm{CH}_{2} \mathrm{OCH}_{2}$ - occur, which rearrange with relative ease to methylene $-\mathrm{CH}_{2}$ - bridges with liberation of formaldehyde. The second step of this rearrangement corresponds to the subsequent reaction of the liberated formaldehyde with the phenolic nuclei of the tannin and does not subtract but reinforces the viscosity increase action of the polycondensation. The time values where both effects occur correspond to the times obtained for these effects through different analysis routes, [12] indicating that this effect does at least contribute to the trend observed.

\section{DETERMINATION OF THE GEL POINT}

During the curing process of Tannin-formaldehyde1 (TF) resins, a network structure is formed and the adhesive passes through different physical and relaxation states from the viscous to the rubbery or even glassy state. The transition from the liquid to solid state occurs at the gel point. Before the gel 
point, the zero shear viscosity can characterize the behavior of the liquid resin. The value of this parameter increases as the polycondensation reaction proceeds, and at the gel point the weight average molecular mass becomes theoretically infinite.

This method relies on the tendency of $M \longrightarrow \alpha$; hence, viscosity $\longrightarrow \alpha$ at the gel point. The method consists then to extrapolate to $\alpha$ values of the viscosity at zero frequency $\left(\eta_{0} \rightarrow \alpha\right.$ or, better for practical reasons, $1 / \eta_{0} \rightarrow 0$ ). The values of the zero frequency viscosity used in this method are those obtained by extrapolating to zero frequency the parameters measured at 2, 4, 6, 8, and $10 \mathrm{rad} / \mathrm{s}$. The application of this method for the case of the reaction of the tannin extract with $5 \%$ paraformaldehyde is shown in Figure 3. The gel point (Fig. 3) is defined as the intersection with the $x$-axis of the prolongation of the linear part of the regression.

\section{$1 / \eta_{0}$ Vs time}

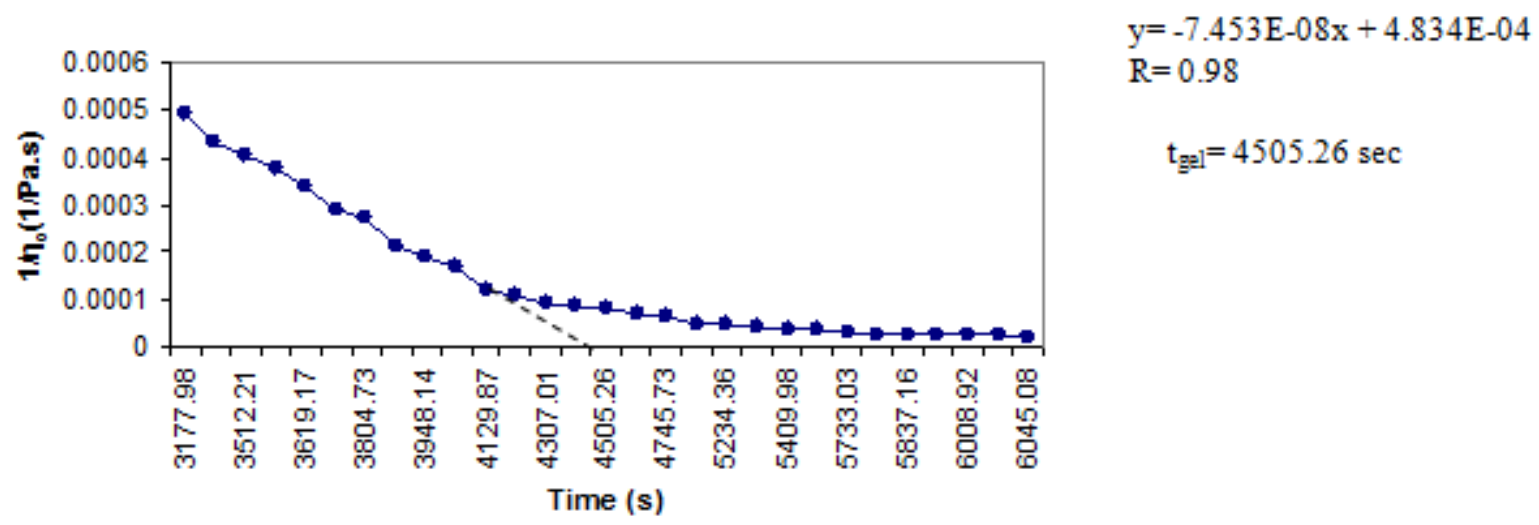

Figure3. The method $\left(/ 1 / \eta_{0} \rightarrow 0\right)$ for the determination of the gel point. Variation of $1 / \eta_{0}$ as a function of time of the system tannin extract $+5 \%$ paraformaldehyde during curing at $65{ }^{\circ} \mathrm{C}$

\section{CONClusions}

The reaction of the polyflavanoid tannin extract of the flower buds of Terminalia chebula with formaldehyde was studied by cone-plate rheometry before the gel point. By following the variation of zero-frequency viscosity as a function of the reaction time, it can be noticed that, before reaching the gel point, three well distinct phases of the reaction can be noticed.

The gel point can be determined by the extrapolation to $\alpha$ of the zero frequency viscosity.

\section{ACKNOWLEDGMENT}

Financial support provided by the National Science Foundation (NSF) of Sri Lanka (Grant No.: RG/ 2006/EB/ 04) is gratefully acknowledged.

\section{REFERENCES}

[1] Pizzi, A. In Wood Adhesives, Chemistry and Technology; Pizzi, A., Ed.; Marcel Dekker: New York, 1983; Vol. 1, Chapter 4.

[2] Colleri, L. Le Fabbriche Italiane di Estratto di Castagno; Milanostampa S.p.A.: Farigliano (CN), Italy, 1989.

[3] Garcia, R.; Pizzi, A. J Appl Polym Sci 1998, 70, 1083.

[4] Garcia, R.; Pizzi, A. J Appl Polym Sci 1998, 70, 1093.

[5] Garcia, R.; Pizzi, A.; Merlin, A. J Appl Polym Sci 1997, 65, 2623.

[6] Garnier, S.; Pizzi, A.; Vorster, O. C.; Halasz, L. J Appl Polym Sci 2001, 81,1634.

[7] Vinogradov, G. V.; Malkin, A. Y. Rheology of Polymers; Springer- Verlag: Berlin, 1980.

[8] Malkin, A. Y.; Kulichikhin, S. G. Adv Polym Sci 1991, 101, 217-256.

[9] Naroditskaya, E. Y.; Khodzhaeva, I. D.; Kulichikhin, S. G.; Pozdnyakou, V. Y.; Yunitskii, I. N., Kireev, V. V.; Malkin, A. Y. Vysikomolek Soed B 1985, 27, 713.

[10] Malkin, A. Y.; Kulichikhin, S. G.; Kozhina, V. A.; Bolotina, L. M. Vysikomolek Soed A 1987, $29,418$. 
Study of Rheology of Tannin Obtained from Flower Buds of Terminalia chebula (Aralu) and Formaldehyde Reaction

[11] Kulichikhin, S. G.; Demina, G. I.; Bokareva, E. Z.; Malkin, A. Y. Plastmassy 1980,1, 57.

[12] Rossouw, D. duT.; Pizzi, A.; McGillivray, G. J Polym Sci Chem Ed 1980, 18, 3323.

Citation: S Arasaretnam. "Study of Rheology of Tannin Obtained from Flower Buds of Terminalia chebula (Aralu) and Formaldehyde Reaction" International Journal of Advan ced Research in Chemical Science (IJARCS), vol. 6, no. 1, pp. 1-5, 2019. http://dx.doi.org/10.20431/2349-0403.0601001

Copyright: (C) 2019 Authors. This is an open-access article distributed under the terms of the Creative Commons Attribution License, which permits unrestricted use, distribution, and reproduction in any medium, provided the original author and source are credited. 